\title{
Herbal Formula Modified Bu-Shen-Huo-Xue Decoction Attenuates Intervertebral Disc Degeneration via Regulating Inflammation and Oxidative Stress
}

\author{
Jialiang Lin $\mathbb{C D}^{1,2,3}$ Jionghui Gu ${ }^{\mathbb{D}},{ }^{4}$ Dongwei Fan $\mathbb{D}^{\mathrm{D}},{ }^{1,2,3}$ and Weishi Li $\mathbb{D}^{1,2,3}$ \\ ${ }^{1}$ Department of Orthopaedics, Peking University Third Hospital, Beijing 100191, China \\ ${ }^{2}$ Beijing Key Laboratory of Spinal Disease Research, Beijing, China \\ ${ }^{3}$ Engineering Research Center of Bone and Joint Precision Medicine, Ministry of Education, Beijing, China \\ ${ }^{4}$ Department of Ultrasound, The First Affiliated Hospital, College of Medicine, Zhejiang University, Hangzhou 310003, China
}

Correspondence should be addressed to Dongwei Fan; fdw@bjmu.edu.cn and Weishi Li; puh3liweishi@163.com

Received 18 August 2021; Revised 15 November 2021; Accepted 6 December 2021; Published 2 February 2022

Academic Editor: Rohit Sharma

Copyright (c) 2022 Jialiang Lin et al. This is an open access article distributed under the Creative Commons Attribution License, which permits unrestricted use, distribution, and reproduction in any medium, provided the original work is properly cited.

\begin{abstract}
Objective. This study aims to clarify the potential mechanism of modified Bu-Shen-Huo-Xue decoction (MBSHXD) in treating intervertebral disc degeneration (IDD) with methods of network pharmacology and molecular docking. Methods. An MBSHXD and IDD-related common target gene set was established through TCMSP, UniProt, and two disease gene databases. GO and KEGG enrichment analysis and protein-protein interaction (PPI) networks were performed through the R platform and STRING to discover the potential mechanism. Molecular docking between the active ingredients and the core genes is used to calculate the binding energy. Results. A total of 147 active ingredients and 79 common genes (including 10 core genes, TNF, VEGFA, IL6, MAPK3, AKT1, MAPK8, TP53, JUN, MMP9, and CXCL8) were identified. The results of GO and KEGG enrichment analysis showed that MBSHXD plays an essential role in regulating inflammation and oxidative stress. The meaningful pathways are the AGE-RAGE signaling pathway in diabetic complications, the IL-17 signaling pathway, the TNF signaling pathway, the PI3K-Akt signaling pathway, the MAPK signaling pathway, and apoptosis. In addition, the PPI network and molecular docking further demonstrated the roles that nine bioactive ingredients of MBSHXD play in IDD treatment through their interference with core target proteins. Conclusion. This study reveals that MBSHXD has the characteristics of a "multi-component, multi-target, and multi-pathway" in the treatment of IDD by regulating inflammation and oxidative stress, and network pharmacology may provide a feasible method to verify the molecular mechanism of MBSHXD for IDD by combining with molecular docking.
\end{abstract}

\section{Introduction}

As a common symptom, low back pain is the leading cause of years living with disability [1]. It is reported that up to $40 \%$ of low back pain is associated with intervertebral disc degeneration (IDD) [2]. IDD is a highly prevalent degenerative spinal disorder that manifests as pain, numbness, and even paralysis of the lower limbs. The discomfort caused by IDD brings great pain and inconvenience to life and work of patients. However, except for nonsteroidal anti-inflammatory drugs (NSAIDs) to relieve acute pain, no effective drugs against IDD are currently available.
In recent years, traditional Chinese medicine (TCM), as an essential branch of complementary and alternative medicine, has received increasing attention for its role in various diseases [3-5]. Modified Bu-Shen-Huo-Xue decoction (MBSHXD), which adds or reduces several herbs based on Bu-Shen-Huo-Xue decoction, is a traditional Chinese herbal recipe that was widely used in elderly patients for fractures therapy in ancient times. MBSHXD consists of the following 9 medicinal herbs: Radix Rehmanniae Preparata, Cortex Eucommiae, Radix Aconiti Lateralis Preparata, Fructus Lycii, Fructus Corni, Semen Persicae, Flos Carthami, Rhizoma Dioscoreae, and Radix Glycyrrhizae. 
According to TCM, MBSHXD has the effects of nourishing the kidney, promoting blood circulation, and strengthening the bones. Recent studies have shown that MBSHXD may delay the progression of degenerative diseases of the musculoskeletal system, including osteoarthritis and IDD [6, 7]. Zhu et al. [7] reported that MBSHXD could inhibit the calcification of the cartilage endplate in gerbils during the process of aging. However, its underlying mechanisms are still unclear.

Network pharmacology, an emerging and interdisciplinary research approach, is based on traditional pharmacology, bioinformatics, chemoinformatics, and network biology $[8,9]$. The development of network pharmacology promotes the transition from the era that a drug fits into the specific target to the generation of mapping the polypharmacology network onto the human disease-gene network [10-12]. Due to the complexity of its composition and function, the clinical application of TCM is distinctly hampered. Fortunately, network pharmacology could be a valuable tool for analyzing and constructing a "drug-targetdisease" interaction network to reveal the molecular mechanisms of action between mutiherbs and the disease [13-15]. Recent studies have confirmed that network pharmacology can help develop TCM applications for the treatment of other diseases or the development of new drugs [16-19] and provide biochemical principles to manage current pandemic diseases, such as COVID-19 [20]. This study aims to clarify the potential mechanism of MBSHXD in the treatment of IDD. The flowchart of our research is shown in Figure 1.

\section{Materials and Methods}

2.1. Active Ingredients and Target Genes in MBSHXD. We got the active ingredients of each herb in MBSHXD through the Traditional Chinese Medicine Systems Pharmacology (TCMSP) database (https://tsmspw.com) [21]. The oral bioavailability $(\mathrm{OB}) \geq 30 \%$ and drug-likeness $(\mathrm{DL}) \geq 0.18$ were set as filtration conditions. Next, the potential target proteins of the selected active ingredients were mined in the DrugBank database (http://www.drugbank.ca) [22].Then, we utilized the UniProt database (https://www.uniprot.org/) [23] to obtain the unique corresponding gene names and to establish the MBSHXD target gene set.

2.2. The Related Target of IDD. IDD-related targets were obtained through retrieving GeneCards (https://www. genecards.org) [24] and Online Mendelian Inheritance in Man (OMIM) (https://omim.org, update November 25, 2020) [25] using the keywords "intervertebral disc degeneration." GeneCards is a comprehensive, user-friendly database that provides information of all annotated and predicted human genes, and we screened for targets with a relevance score $\geq 10$ [26]. The OMIM database provides an organized description of human genes and phenotypes and the relationships between them and is a comprehensive, authoritative, and timely resource for research [25]. Finally, the results of the two databases were summarized, integrated, and deduplicated, and then 1595 potential targets were obtained, and all targets were standardized in the UniProt database (https://www.uniprot.org/) [23].

2.3. Network Visualization and Enrichment Analysis. A common gene set between MBSHXD targets and IDD-related genes was built by the Venn diagram. According to a common gene set, we identified the active components corresponding to each gene and constructed an "active ingredient-common target gene network" using Cytoscape 3.7.2 software [27]. In addition, we have established a "disease-core genes-active ingredients-herbs" network.

The biological functions of genes were revealed in three different aspects, including biological processes (BPs), cell components (CCs), and molecular functions (MFs), by gene ontology (GO) enrichment analysis. The Kyoto Encyclopedia of Genes and Genomes (KEGG) is a database resource for understanding high-level functions and utilities of the biological system, such as cells, organisms, and ecosystems, from molecular-level information. We used the clusterProfiler package of the $\mathrm{R}$ platform for GO and KEGG functional enrichment analysis [28].

\subsection{Protein-Protein Interaction (PPI) Network Construction} and Core Gene Selection. The common genes of MBSHXD targets and IDD-related genes were used to build a PPI network through the STRING database (https://www.string$\mathrm{db} . o r g /)$ [29]. In this database, we set the organism as "Homo sapiens" and a confidence score $>0.4$. In the same way, the PPI network of IDD targets was also established. The PPI network of the common target was imported into the Cytoscape software to make it visible. A cytoHubba plugin [30] was used to identify the top 10 core genes by a method of maximal clique centrality (MCC). In addition, the cluster analysis was performed for the IDD targets PPI network by using the MCODE plugin in Cytoscape software [31]. The parameter conditions are as follows: a node score cutoff $=0.2, \mathrm{k}$ core $=2$, maximum depth $=100$, and a degree cutoff $=2[32]$.

2.5. Molecular Docking. The 3D structures of the proteins encoded by the core genes and the $2 \mathrm{D}$ structures of active ingredients were downloaded from the RSCB PDB database (https://www.rcsb.org/) and PubChem database (https:// pubchem.ncbi.nlm.nih.gov/), respectively. The energy of the molecular ligand was optimized using the MM2 calculation method and derived using the ChemBio 3D software. The receptor protein was dehydrated and smallmolecule ligand was removed with PyMOL 2.4.0 software. The AutoDock tool performed hydrogenation of proteins and active ingredients. Finally, AutoDock Vina was used to calculate the binding energy between the receptor protein and active components [33].

\section{Result}

3.1. Screening of Candidate Targets and Active Ingredients. The MBSHXD contained the following 9 main herbs: Radix Rehmanniae Preparate (Shudihuang), Cortex Eucommiae 


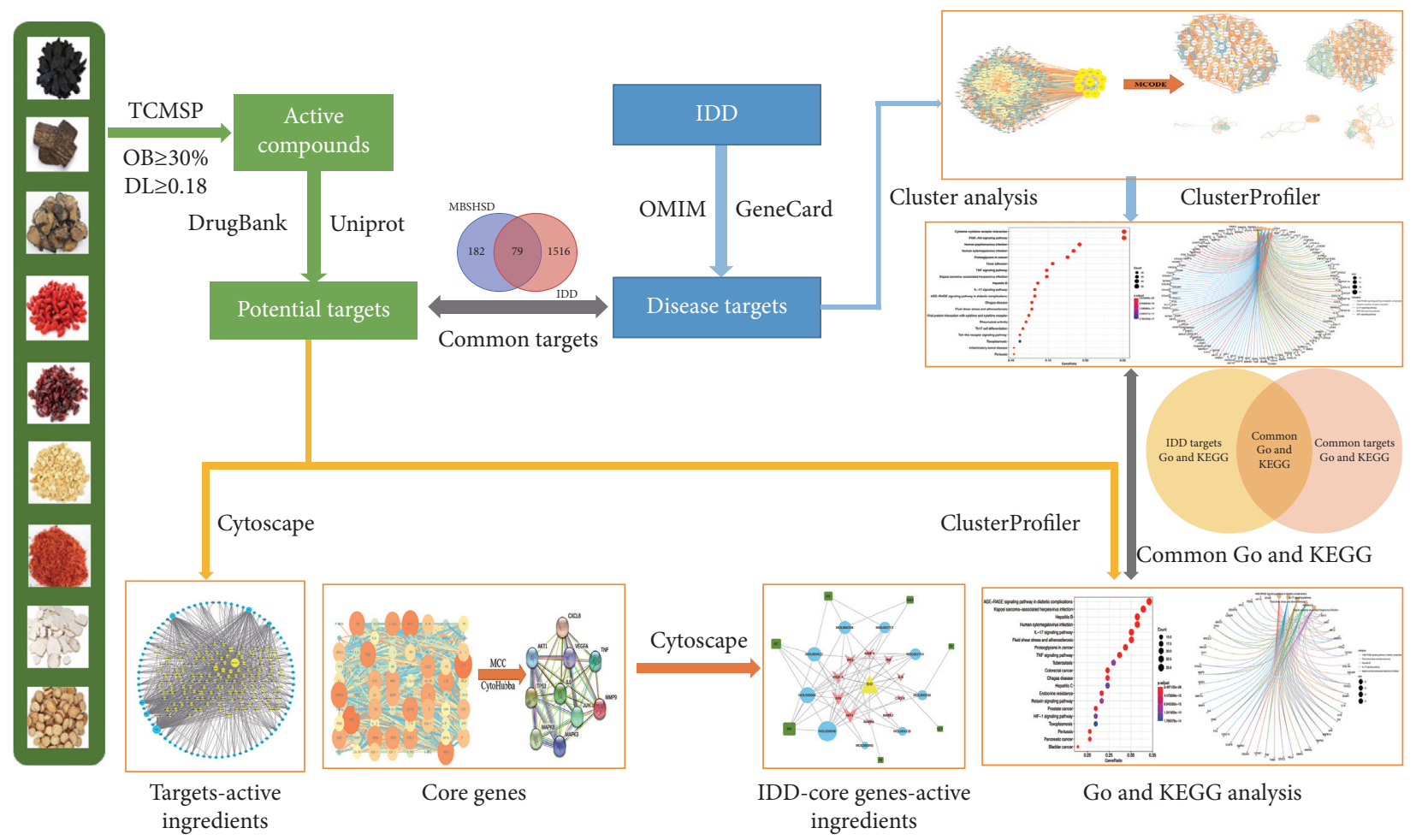

Figure 1: Flowchart to explore the possible mechanism of modified Bushen Huoxue decoction against intervertebral disc degeneration (IDD).

(Duzhong), Radix Aconiti Lateralis Preparate (Fuzi), Fructus Lycii (Gouqizi), Fructus Corni (Shanzhuyu), Semen Persicae (Taoren), Flos Carthami (Honghua), Rhizoma Dioscoreae (Shangyao), and Radix Glycyrrhiza (Ganzao). A total of 244 active ingredients of MBSHXD were obtained through searching TCMSP (Supplementary Table 1). Based on the active ingredients, we acquired 299 targets, which were protein names coded by genes (Supplementary Table 2). Then, after converting the gene symbols by the UniProt database, 261 drug targets were gained (Supplementary Table 3). In addition, a total of 1595 IDD-related genes were obtained from the two abovementioned disease gene databases. By intersecting the targets of MBSHXD and IDD, 79 common target genes were acquired, corresponding to which there were 147 active components (Supplementary Table 4, Figure 2(a)).

3.2. Common Targets-Active Ingredients Network. A network of common targets-active ingredients with 226 nodes and 904 edges was visualized using Cytoscape software (Figure 2(b)).

3.3. PPI Network Construction of IDD Target. A proteinprotein interaction network was established using the STRING database, which showed complex relationships between the proteins encoded by IDD genes (Figure 3). We selected 10 genes (AKT1, IL6, TP53, VEGFA, TNF, FN1, EGFR, EGF, MAPK3, and MYC) whose largest degree was demonstrated on the right. The PPI network was imported into the Cytoscape software for further cluster analysis and visualization with the MCODE plugin. Finally, the top five clusters were selected from 32 clusters based on their MCODE score (Figure 3 and Table 1). In addition, we performed GO functional enrichment and KEGG pathway analysis on the IDD target genes contained in these 5 clusters. Among the 291 genes, we acquired 3088 BPs, $93 \mathrm{CCs}, 135 \mathrm{MFs}$, and 151 KEGG pathways with $P$ value less than or equal to 0.05 (Supplementary Table 5). Then, Figure 4 shows the top 10 for GO functional enrichment and the top 20 for KEGG pathway analysis.

The biological processes of IDD may be mainly related to the inflammatory response and the exogenous pathways of apoptosis, such as leukocyte migration, extracellular matrix (ECM) organization, extracellular structure organization, and extrinsic apoptotic signaling pathways. The molecular functions of IDD would be related to cytokine activity, cytokine receptor binding, and signaling receptor activator activity. The cellular components associated with IDD might activate the collagen-containing ECM, endoplasmic reticulum lumen, and external side of the plasma membrane (Figure 4). Meanwhile, the results of KEGG indicated that the main pathways were related to cytokine-cytokine receptor interaction, the PI3K-Akt signaling pathway, and the TNF signaling pathway (Figure 4).

3.4. Enrichment Analysis of Common Genes. To further explore the interactions between common target genes and the mechanisms by which MBSHXD may treat IDD, GO enrichment analysis and KEGG pathway enrichment were performed using the R platform. We acquired a total of 2114 


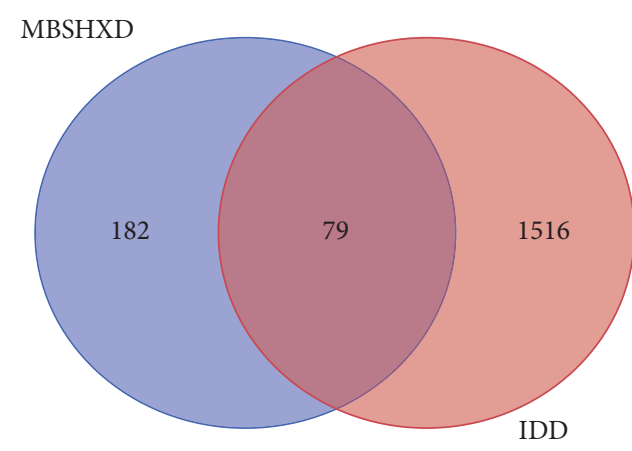

(a)

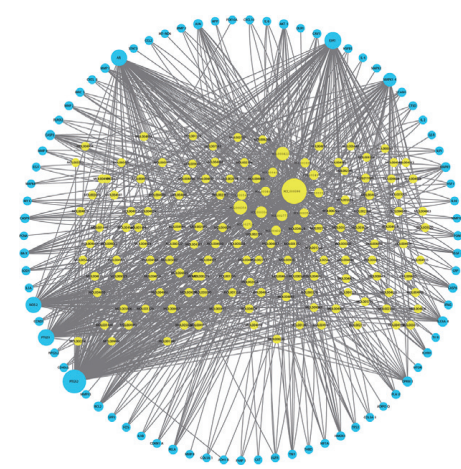

(b)

FIGURE 2: Construction of common gene-active ingredients network. (a) Venn diagram. (b) Common targets-active ingredients network. Yellow nodes represent the common targets of IDD and modified Bushen Huoxue decoction; blue nodes represent the active ingredients related to the common targets. The line between two nodes represents the interaction; the size of each node represents the number of connections.
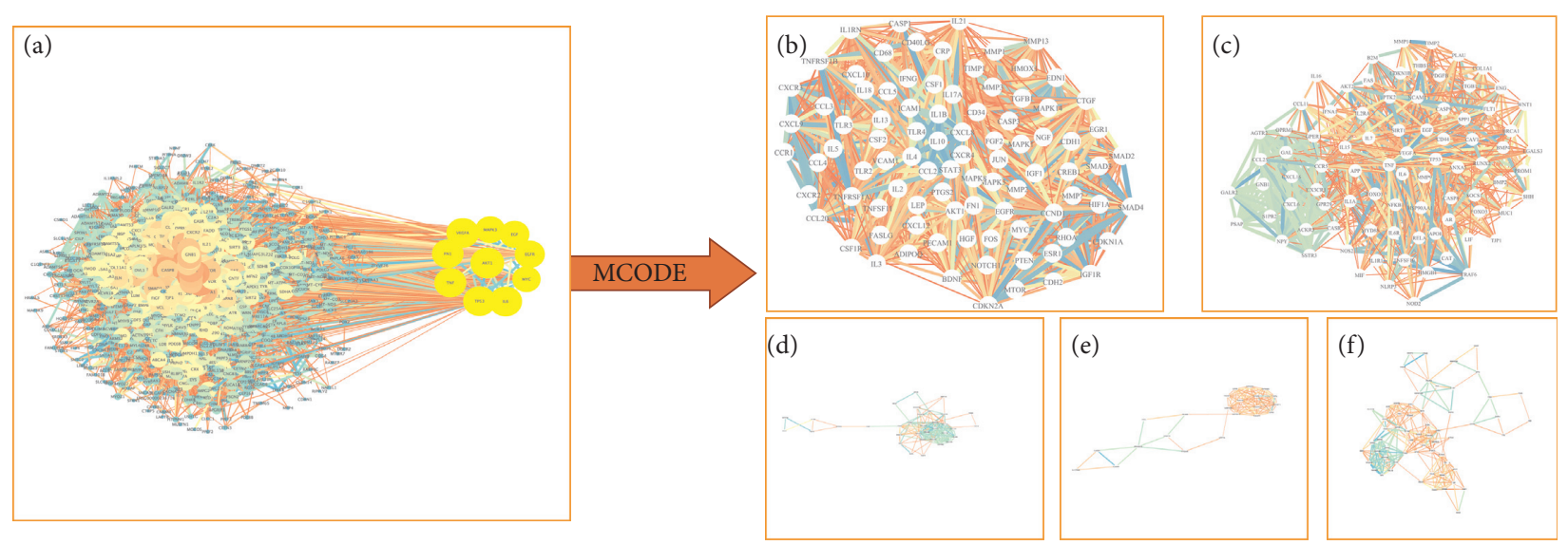

FIgURE 3: Protein-protein interaction (PPI) network and cluster analysis of the IDD targets. (a) PPI network of IDD targets. The 10 genes with the largest degree values are shown on the right with yellow nodes. (b-f) The top 5 cluster graphs of the IDD targets from its PPI network.

TABLE 1: The top 5 cluster information of the IDD targets from its network

\begin{tabular}{lccc}
\hline Cluster & Score & Nodes & Edges \\
\hline 1 & 61.506 & 90 & 2737 \\
2 & 23.952 & 84 & 994 \\
3 & 9.091 & 34 & 150 \\
4 & 8.762 & 22 & 92 \\
5 & 8.4 & 61 & 252 \\
\hline
\end{tabular}

entries about biological processes. The top 10 are shown in Figure 5, among which 4 items were related to oxidative stress. By analyzing the results of the cellular component, we found that the target genes mainly acted in the membrane raft, membrane microdomain, and membrane region. The main molecular functions of common targets were cytokine activity, cytokine receptor binding, signaling receptor activator activity, and receptor-ligand activity. Simultaneously, the top 20 pathways were selected to investigate the possible mechanism of MBSHXD on the disease (Figure 5). The most meaningful pathways of common targets are the AGERAGE signaling pathway in diabetic complications, the TNF signaling pathway, and apoptosis. All the results of the enrichment analysis are listed in Supplementary Table 6.

3.5. PPI Network of Common Target and Core Genes. Firstly, we inputted 79 common genes into the STRING to construct the PPI network (Figure 6). To further obtain the core genes of the MBSHXD treatment for IDD, the obtained PPI network was imported into the software through the cytoHubba plugin with the MCC method. Finally, the top 10 genes were identified as core genes (TNF, VEGFA, IL6, MAPK3, AKT1, MAPK8, TP53, JUN, MMP9, and CXCL8). Fortunately, there are six genes (TNF, VEGFA, IL6, MAPK3, AKT1, and TP53) that overlap with the critical genes for IDD. In addition, we found that the PPI network for 10 core genes all had a node degree of 9 (Figure 6).

3.6. Selection of Critical Enrichment Analysis. After comparing and analyzing the KEGG enrichment of the disease targets and common targets of MBSHXD and IDD, we found 138 overlapping KEGG pathways (Supplementary 


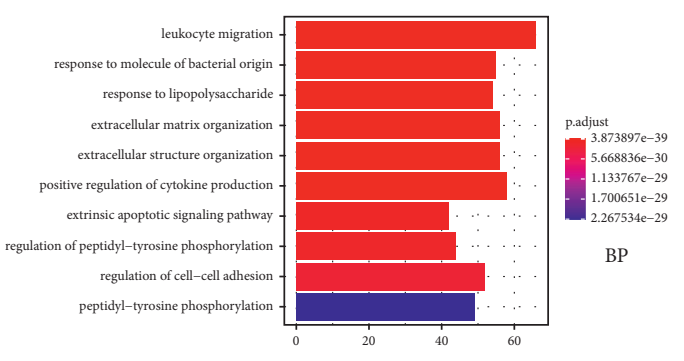

(a)

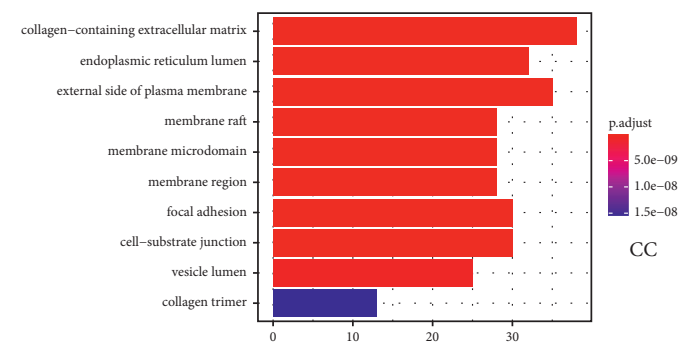

(c)

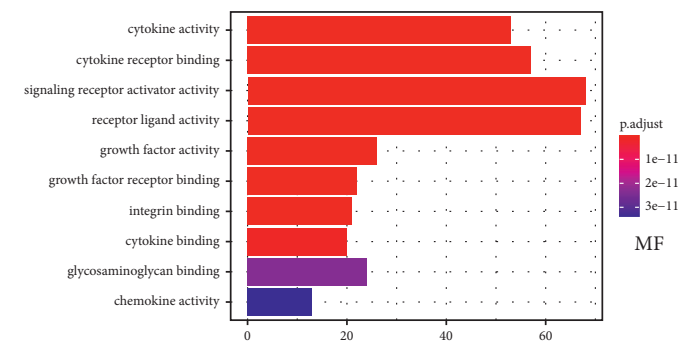

(e)

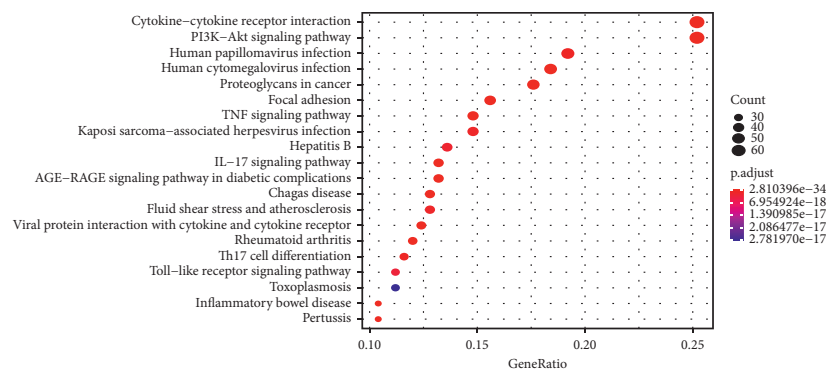

(g)

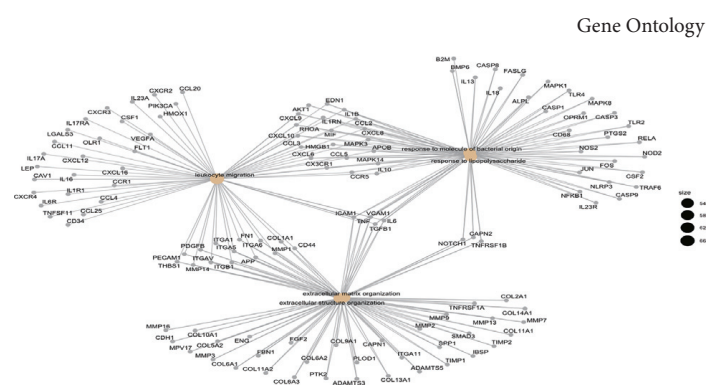

(b)

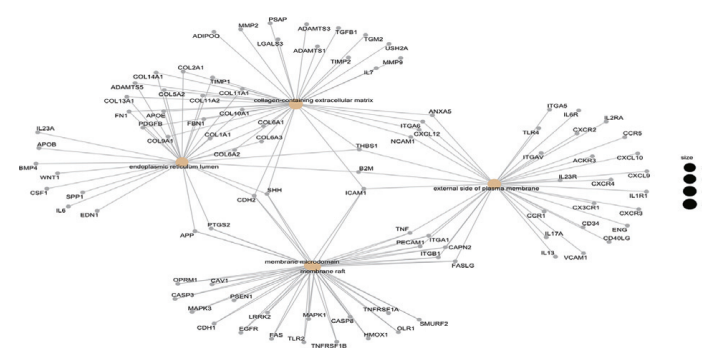

(d)

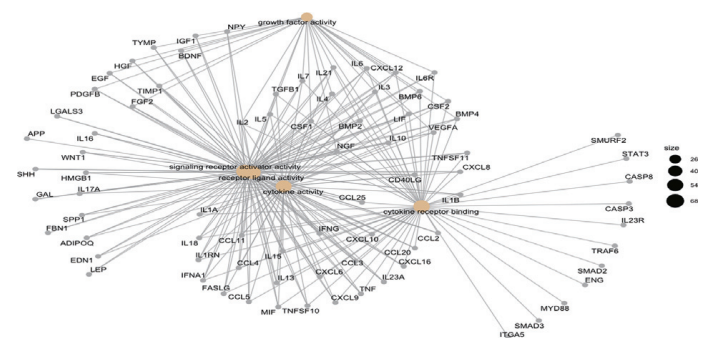

(f)

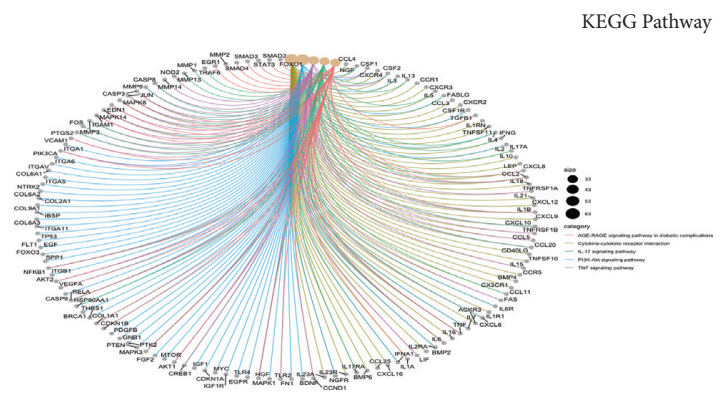

(h)

Figure 4: GO (including BP, MF, and CC) and KEGG analysis of IDD-related genes. (a, c, d) The top 10 significantly GO (BP, MF, and CC) enriched terms are arranged according to the adjusted $P$ value. (b, e, f) Subnetwork showing the top five terms and related genes. ( $g$ ) The 20 pathways with the lowest adjusted $P$ values. The darker the color, the smaller the adjusted $P$ value. The larger the circle, the greater the number of target genes in the term. (h) Subnetwork showing the top five KEGG pathways and related genes.

Table 7). To further screen these pathways, we sorted them according to the proportion of genes and compared the potentially related IDD pathways in PubMed with the abovementioned ways. Finally, 9 relatively related IDD pathways were obtained. Table 2 shows the basic information of the 9 pathways that may be relevant for IDD.

3.7. Disease-Core Genes-Active Ingredients-Herbs Network. According to the 10 core genes, we found the corresponding seven herbs and nine active components. The disease-core genes-active ingredients-herbs network is demonstrated in Figure 7 . The 9 active components are sorted by degree as follows: quercetin (MOL000098), luteolin (MOL000006), kaempferol (MOL000422), beta-sitosterol (MOL000358), baicalein (MOL002714), beta-carotene (MOL002773), diosgenin (MOL000546), naringenin (MOL004328), and formononetin (MOL000392). The basic information of the nine active components in MBSHXD is listed in Table 3. Quercetin (MOL000098) was the essential active compound with the largest degree, which may play a key role in IDD treatment by MBSHXD. 


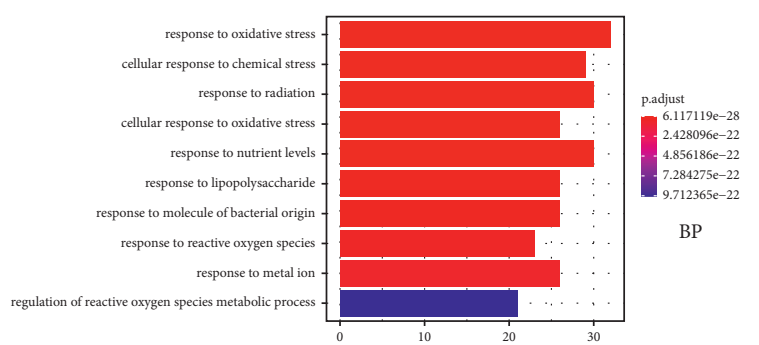

(a)

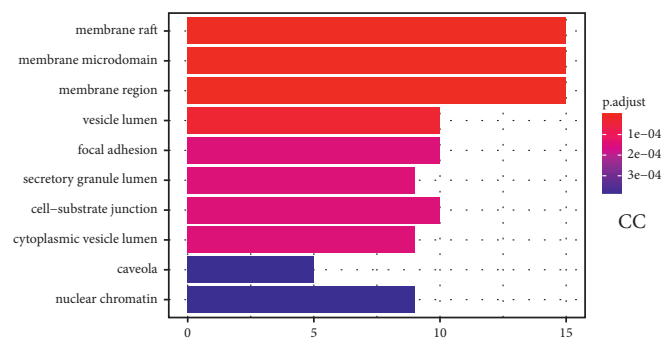

(c)

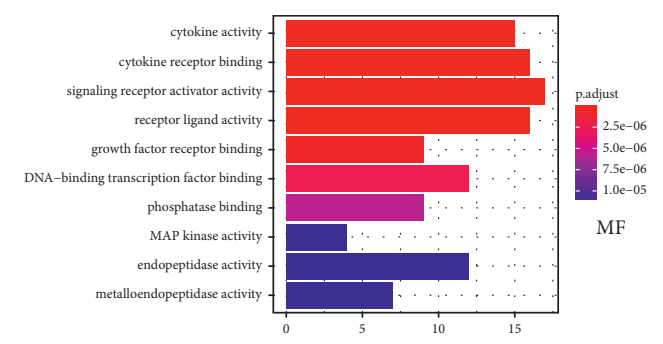

(e)

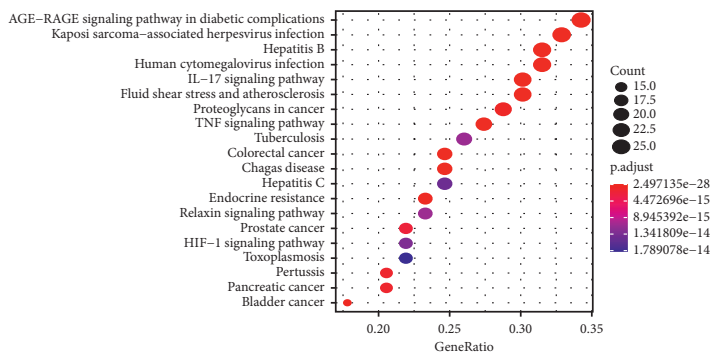

(g)

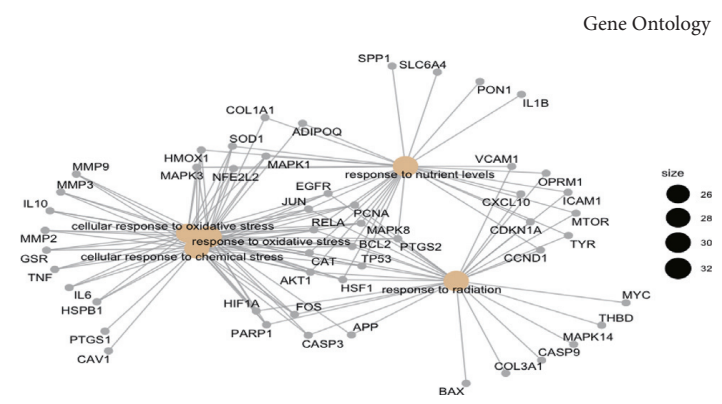

(b)

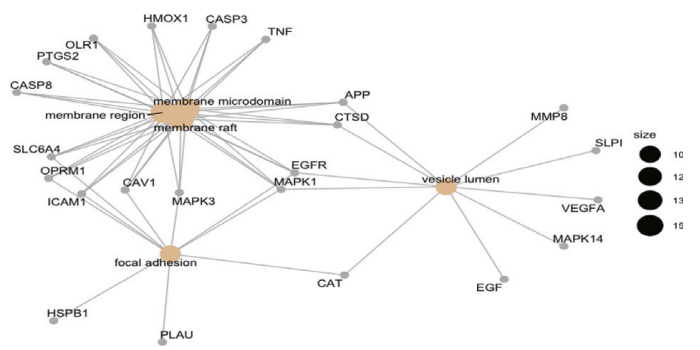

(d)

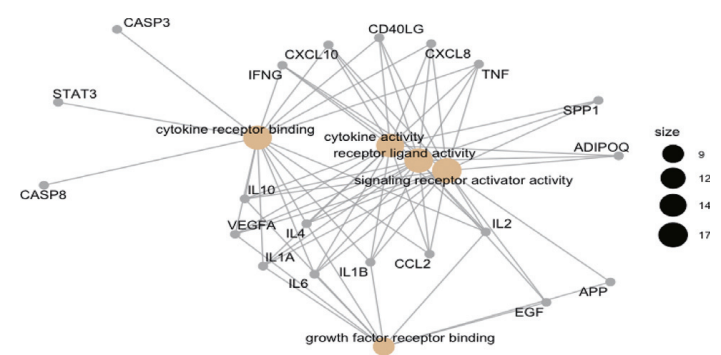

(f)

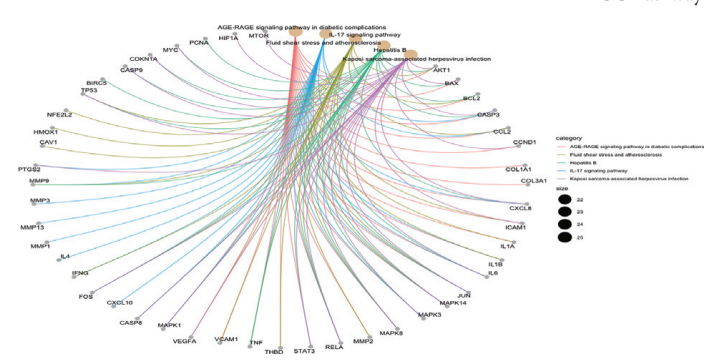

(h)

FIgure 5: GO (including BP, MF, and CC) and KEGG analysis of common genes. (a, c, d) The top 10 significantly GO (BP, MF, and CC) enriched terms are arranged according to the adjusted $P$ value. (b, e, f) Subnetwork showing the top five terms and related genes. ( $g$ ) The 20 pathways with the lowest adjusted $P$ values. The darker the color, the smaller the adjusted $P$ value. The larger the circle, the greater the number of target genes in the term. (h) Subnetwork showing the top five KEGG pathways and related genes.

\subsection{Molecular Docking Verified Active Ingredients and Core} Genes Encoding Proteins. Ten key genes were used as receptor proteins, and nine active compounds were used as ligands for molecular docking verification. The details of 10 receptor proteins are listed in Table 4, and Figure 8 shows these results [34]. Binding energy, which is the criterion to judge stability, less than $-5.0 \mathrm{kcal} / \mathrm{mol}$ was considered relatively stable between proteins and small-molecule compounds. As we expected, the binding energies between the active ingredients and the core genes were all less than $-5.0 \mathrm{kcal} / \mathrm{mol}$, indicating that all active compounds can easily enter the active pocket of proteins coded by core genes and bind stably. We selected 4 proteins and small molecules with the highest binding energy for demonstration (Figure 9).

\section{Discussion}

IDD is a widespread degenerative disease of the spine. With the increasing aging of society, the incidence of IDD has been on the rise in recent years and tends to be younger. The incidence rate of cervical, thoracic, and lumbar diseases 


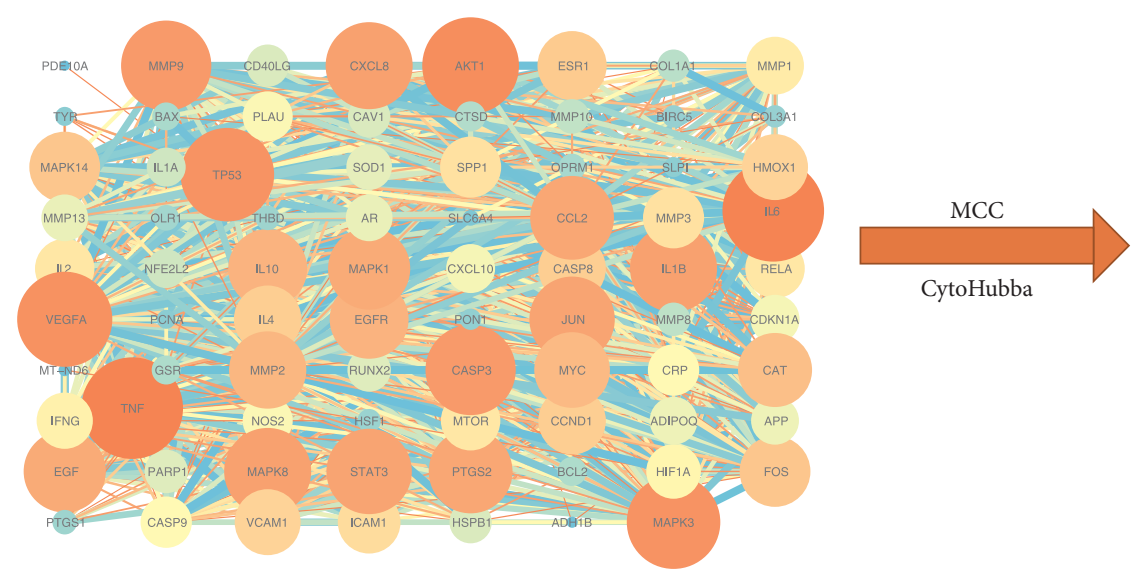

(a)

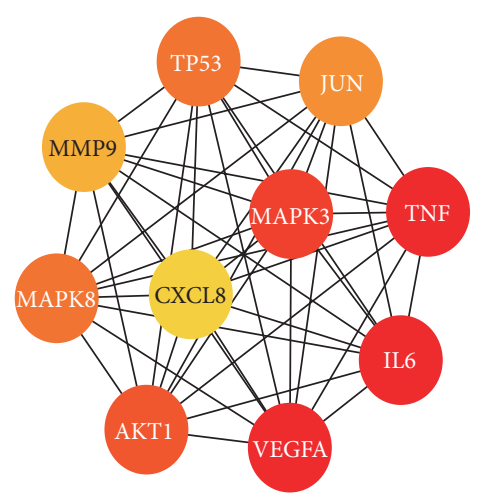

(b)

FIGURE 6: Confirmation of core genes. (a) Visualization of seventy-nine common targets protein-protein interaction (PPI) network with 79 nodes and 1487 edges. (b) PPI network of the core genes.

TABLE 2: The enriched 9 possible related pathways for IDD.

\begin{tabular}{|c|c|c|c|c|c|c|}
\hline \multirow[t]{2}{*}{ ID } & \multirow[t]{2}{*}{ Description } & \multicolumn{2}{|c|}{$\begin{array}{c}\text { KEGG index } \\
\text { enriched by IDD } \\
\text { targets }\end{array}$} & \multicolumn{3}{|c|}{ KEGG index enriched by common targets } \\
\hline & & p. adjust & Count & p. adjust & Count & Count of active ingredients \\
\hline Hsa04933 & $\begin{array}{l}\text { AGE-RAGE signaling pathway in diabetic } \\
\text { complications }\end{array}$ & $4.08 E-24$ & 33 & $2.4971 E-28$ & 25 & 125 \\
\hline Hsa04657 & IL-17 signaling pathway & $5.06 E-25$ & 33 & $3.4116 E-24$ & 22 & 243 \\
\hline Hsa04668 & TNF signaling pathway & $5.71 E-27$ & 37 & $9.6621 E-20$ & 20 & 245 \\
\hline Hsa04151 & PI3K-Akt signaling pathway & $1.19 E-29$ & 63 & $7.355 E-10$ & 19 & 47 \\
\hline Hsa04210 & Apoptosis & $1.32 E-11$ & 24 & $2.0176 E-14$ & 17 & 66 \\
\hline Hsa04010 & MAPK signaling pathway & $6.74 E-15$ & 40 & $3.2112 E-10$ & 16 & 54 \\
\hline Hsa05206 & MicroRNAs in cancer & $1.83 E-08$ & 31 & $3.389 E-08$ & 16 & 188 \\
\hline Hsa05010 & Alzheimer's disease & $2.69 E-06$ & 30 & $3.3702 E-07$ & 16 & 257 \\
\hline Hsa05215 & Prostate cancer & $6.98 E-17$ & 26 & $2.1985 E-15$ & 16 & 127 \\
\hline
\end{tabular}

caused by IDD is up to $80 \%$, which has become a major global health problem [35]. At present, the interventions for IDD mainly include the use of pain relievers or surgical treatment $[36,37]$. However, there is still a lack of effective nonsurgical treatments for IDD, making the exploration of novel and effective nonsurgical treatments for IDD a focus of research in degenerative spinal disorders. Notably, TCM has been used for more than 2,000 years to treat various diseases, including IDD [38]. TCM mainly focuses on expelling wind and cold, tonifying the liver and kidney in the treatment of IDD, supplemented by tonifying the spleen and replenishing qi, promoting blood circulation, and dredging collaterals [39-41]. MBSHXD, which comes from the ancient Chinese literature "Shangke Dacheng," consists of 9 Chinese herbs, which are as follows: Shudihuang, Duzhong, Fuzi, Gouqi, Shanzhuyu, Taoren, Honghua, Shanyao, and Gancao [42]. It was demonstrated that MBSHXD could promote the proliferation of nucleus pulposus (NP) cells and remodel the ECM during IDD [43]. However, due to the complex composition and comprehensive action of MBSHXD, the specific mechanism of its treatment for IDD is still unclear, so we decided to use an approach of network pharmacology to reveal it and lay a foundation for further studies.
We used Cytoscape software to reconstruct the PPI network of common genes obtained from the STRING database and got 10 core genes (TNF, VEGFA, IL6, MAPK3, AKT1, MAPK8, TP53, JUN, MMP9, and CXCL8) of MBSHXD against IDD by the MCC algorithm. These core target proteins are involved in multiple signaling pathways such as inflammation, immunity, metabolism, and proliferation-related signaling pathways. Studies have confirmed that the process of IDD can be delayed by inhibiting the TNF- $\alpha$-induced inflammatory response and reducing the expression of inflammatory cytokines, including IL-6 $[44,45]$. It is worth noting that TP53 was found to be potentially involved in the progression of IDD by analyzing the microarray datasets of GSE19943, GSE15227, and GSE34095 [46]. Akt1 is a serine/threonine protein kinase that is involved in inflammation and cell metabolism through a wide variety of signaling pathways such as PI3KAkt and MAPK signaling. Wang et al. found that the degeneration of NP cells was ameliorated by regulating the ITGA2/PI3K/Akt signaling pathway [47]. Moreover, Zhan et al. found that the degree of intervertebral disc degeneration was related to the loss of vascular buds and the downregulation of VEGFA and its receptors [48]. 


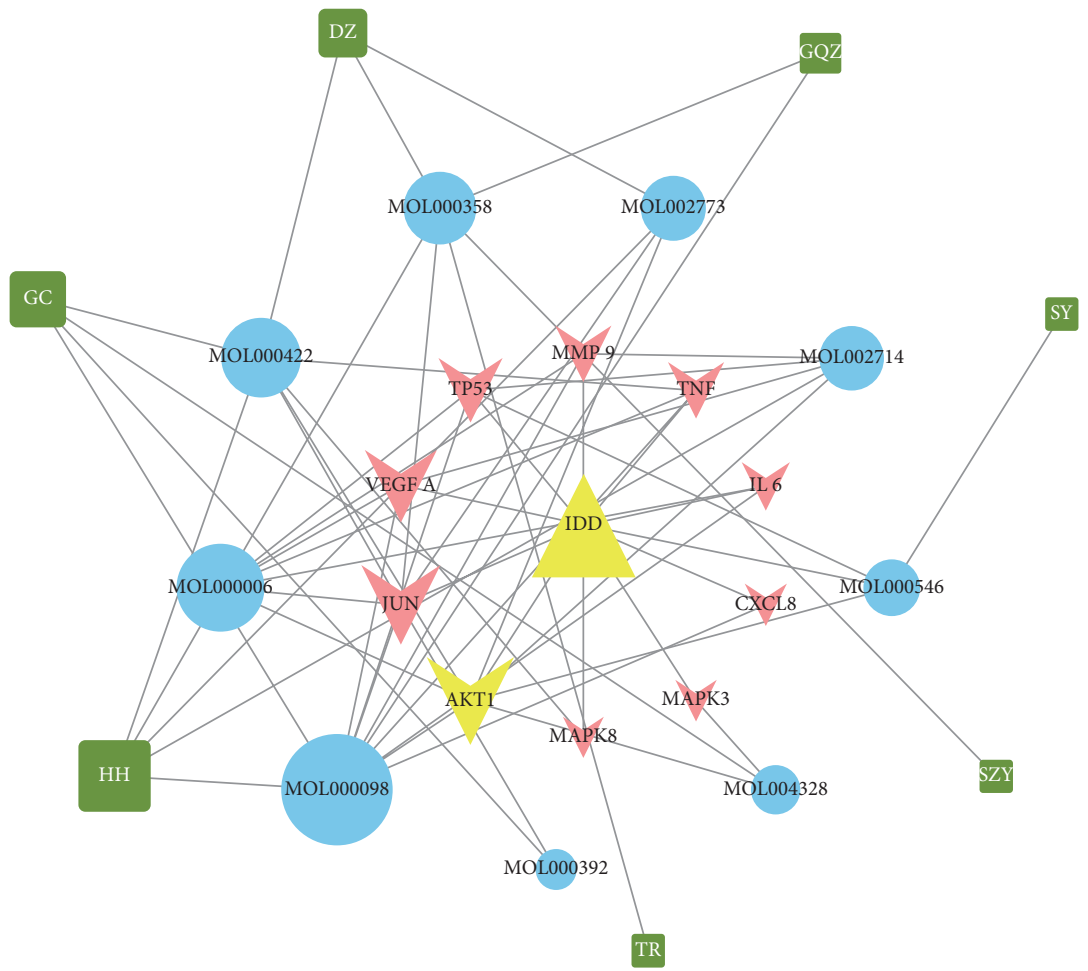

FIGURE 7: IDD-core gene-active ingredient-herb network. Red triangle nodes represent disease, pink arrow-like nodes represent core genes, blue circle nodes represent the active ingredients related to the core genes, and the green square nodes represent herbs. The size of each node was set according to its degree value.

TABLE 3: Basic information of the nine active components in MBSHXD.

\begin{tabular}{|c|c|c|c|c|c|c|}
\hline Molecule ID & Molecule name & PubChem CID & OB $(\%)$ & $\mathrm{DL}$ & Source (herb name) & Targeted core genes \\
\hline MOL000098 & Quercetin & 5280343 & 46.43 & 0.28 & $\begin{array}{l}\text { Duzhong, Gouqizi, Honghua, } \\
\text { Gancao }\end{array}$ & $\begin{array}{c}\text { AKT1, CXCL8, IL6, JUN, MMP9, } \\
\text { TNF, TP53, VEGFA }\end{array}$ \\
\hline MOL000006 & Luteolin & 5280445 & 36.16 & 0.25 & Honghua & $\begin{array}{c}\text { TNF, MMP9, IL6, VEGFA, TP53, } \\
\text { JUN, AKT1 }\end{array}$ \\
\hline MOL000422 & Kaempferol & 5280863 & 41.88 & 0.24 & Duzhong, Honghua, Gancao & TNF, MAPK8, JUN, AKT1 \\
\hline MOL000358 & Beta-sitosterol & 222284 & 26.91 & 0.75 & $\begin{array}{c}\text { Duzhong, Gouqizi, Shanzhuyu, } \\
\text { Taoren, Honghua }\end{array}$ & JUN \\
\hline MOL002714 & Baicalein & 5281605 & 33.52 & 0.21 & Honghua & MMP9, VEGFA, TP53, AKT1 \\
\hline MOL002773 & Beta-carotene & 5280489 & 37.18 & 0.58 & Duzhong, Honghua & VEGFA, JUN, AKT1, \\
\hline MOL000546 & Diosgenin & 99474 & 80.88 & 0.81 & Shanyao & VEGFA, TP53, AKT1 \\
\hline MOL004328 & Naringenin & 932 & 59.29 & 0.21 & Gancao & MAPK3, AKT1 \\
\hline MOL000392 & Formononetin & 5280378 & 69.67 & 0.21 & Gancao & JUN \\
\hline
\end{tabular}

TABLE 4: Detailed information of the 10 core targets.

\begin{tabular}{lcc}
\hline Protein & PDB ID & Relevant citation \\
\hline TNF & 7KP9 & DOI: $10.1038 /$ s41467-020-20828-3 \\
VEGFA & 4DEQ & DOI: $10.1074 /$ jbc.M111.331140 \\
ILA & 1ALU & DOI: $10.1093 /$ emboj/16.5.989 \\
MAPK3 & 6GES & DOI: $10.1016 /$ j.chembiol.2019.02.021 \\
AKT1 & 6S9W & DOI: $10.1002 /$ anie.201909857 \\
MAPK8 & 4L7F & DOI: $10.1016 /$ j.bmcl.2013.06.087 \\
TP53 & 7DHZ & DOI: $10.1016 /$ j.ccell.2020.11.013 \\
JUN & 1JUN & DOI: $10.1074 /$ jbc.271.23.13663 \\
MMP9 & 5CUH & DOI: $10.1016 /$ j.ejmech.2016.01.053 \\
CXCL8 & 1IKL & DOI: $10.1021 /$ bi00040a008 \\
\hline
\end{tabular}

According to our "disease-core genes-active ingredients-herbs" network, nine bioactive compounds between the 10 core genes and the corresponding active compounds in MBSHXD were identified. Furthermore, three hub ingredients, quercetin, luteolin, and kaempferol, were selected, and they targeted the largest number of core genes. In vitro experiments showed that quercetin ameliorated the progression of IDD by suppressing the expression of senescence associated secreted phenotype factors and improving the senescence phenotype of NP cells via the Nrf $2 / \mathrm{NF}-\kappa \mathrm{B}$ axis [49]; luteolin, a natural flavonoid, has anti-inflammatory and anticatabolic effects [50]; kaempferol has been reported 


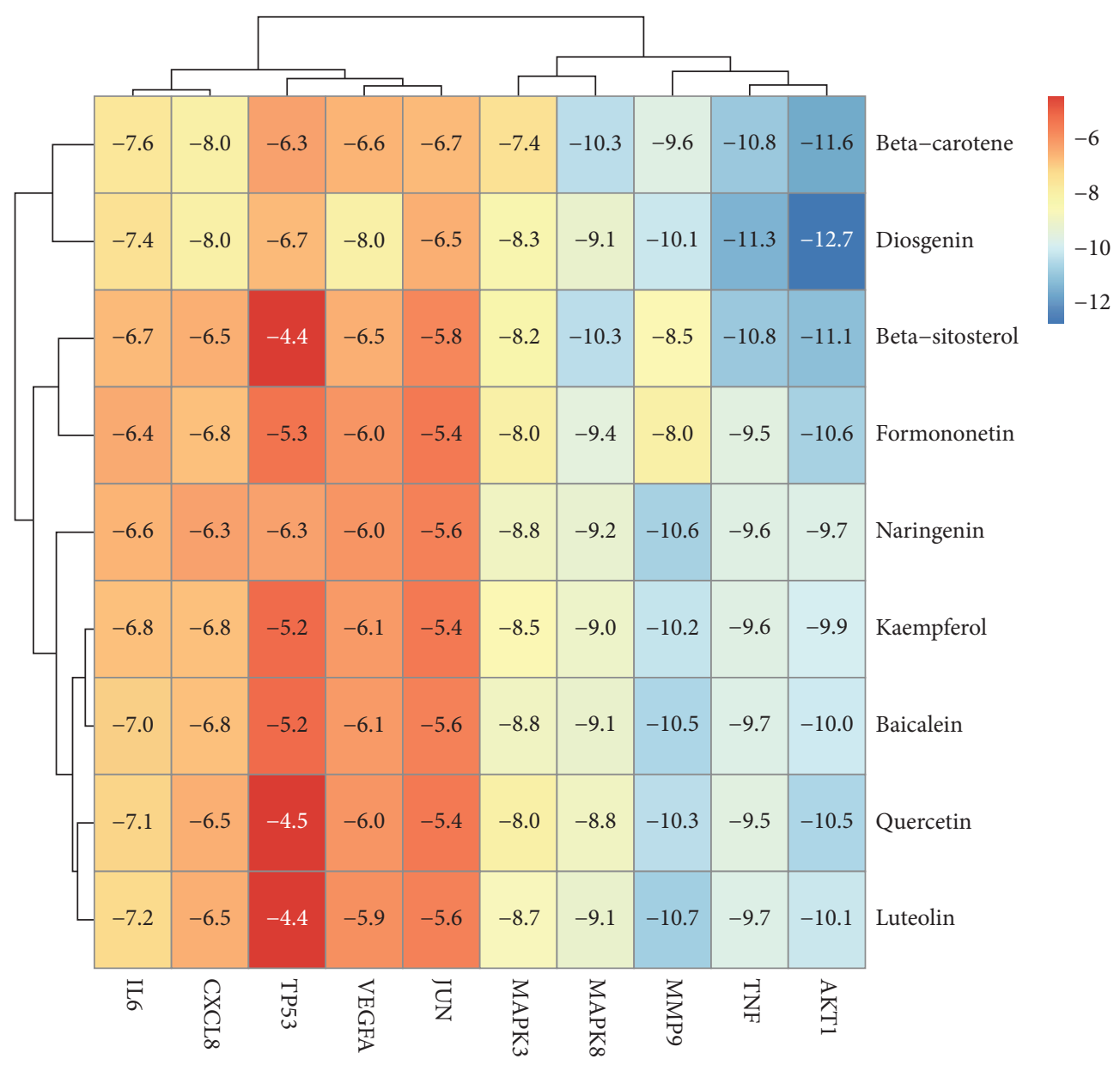

Figure 8: Heat map of molecular docking between 10 core genes and 9 active ingredients [34].

to regulate osteogenesis/adipogenesis balance and inhibit inflammation in bone mesenchymal stem cells to slow the progression of IDD [51]. These compounds are the material basis of MBSHXD in the treatment of IDD.

Based on the ten core genes and their corresponding core compounds, molecular docking was performed to demonstrate the binding energy between proteins and smallmolecule compounds, which all showed good binding affinity. The abovementioned molecular docking results were presented in the form of a heat map by using R software. In addition, the software package ClusterProfiler was used for enrichment analysis of GO and KEGG pathways. The results of two enrichment analyses of GO and KEGG were compared with the IDD-related pathways searched in PubMed. We speculated that the therapeutic effect of MBSHXD in IDD may be related to its anti-inflammatory and antioxidative stress effects, which inhibit apoptosis and senescence of NP cells. Indeed, inflammation, ECM degradation, and senescence of NP cells are thought to be the main pathogenic mechanisms of IDD $[52,53]$. The abovementioned mechanisms mainly involve the AGE-RAGE signaling pathway in diabetic complications, the IL-17 signaling pathway, the TNF signaling pathway, the PI3K-Akt signaling pathway, the MAPK signaling pathway, and apoptosis. It has been reported that the PI3K-Akt pathway is involved in the proliferation, apoptosis, senescence, and ECM metabolism of NP cells and is significantly associated with NP degeneration. It is considered as an essential signaling pathway involved in IDD [54-56]. TNF and IL-17 pathways play a synergistic role in IDD progression, mainly by promoting the release of inflammatory mediators, the apoptosis of NP cells, and the degradation of ECM [57-59]. In addition, the MAPK pathway is thought to primarily mediate the inflammatory response of NP cells to TNF- $\alpha$ stimulation [60]. Illien-Jünger et al. [61] suggested AGE accumulation is related to endochondral ossification, which may induce hypertrophy and osteogenic differentiation of intervertebral disc cells through the AGE/RAGE axis. More importantly, the accumulation of AGEs is closely associated with oxidative stress, which may lead to alterations in the oxidative microenvironment of NP and, in turn, contribute to IDD. Thus, targeted clearance of AGEs may be a promising direction to noninvasively slow down the progression of IDD.

However, our study also has some limitations. For example, the results of this study lacked validation by in vitro experiments, while further external validation in animals should be performed. In addition, the database we selected may not be comprehensive enough, and the active ingredients and related genes screened through the database may have been missed. 


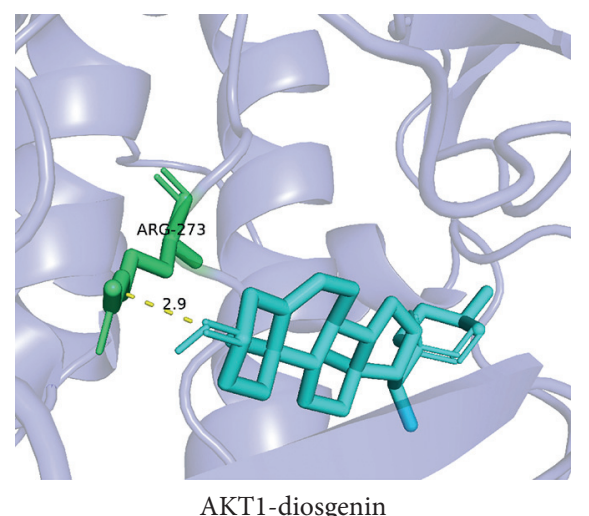

(a)

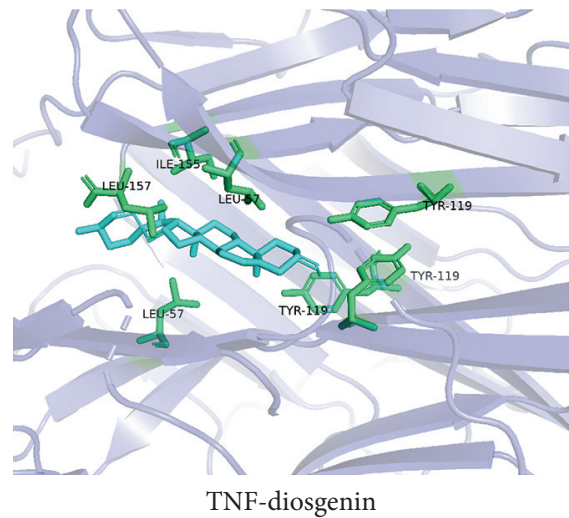

(c)

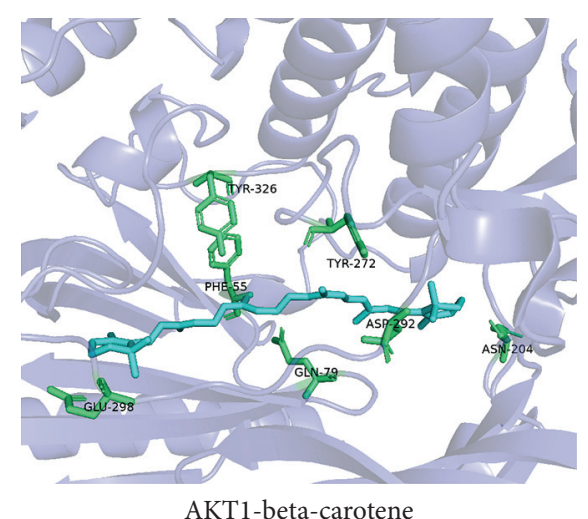

(b)

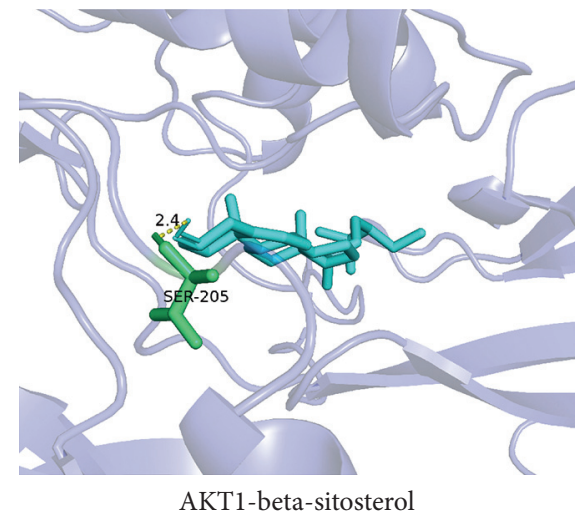

(d)

Figure 9: Molecular docking of "bioactive compound-hub gene." (a) AKT1 to Diosgenin; (c) TNF to Diosgenin; (b) AKT1 to betacarotene; and (d) AKT1 to betasitosterol.

In conclusion, we reveal that MBSHXD has the characteristics of "multi-component, multi-target, and multi-pathway" in IDD treatment based on network pharmacology and molecular docking. MBSHXD retards the progression of IDD by regulating the antioxidant stress and inflammatory response, which inhibits apoptosis and senescence of NP cells. The primary mechanism is related to 9 core bioactive components, 10 core genes, and 9 related pathways. This study may inspire novel treatment strategies for IDD and inform future research.

\section{Data Availability}

The data used to support the findings of this study are included within the article.

\section{Conflicts of Interest}

The authors declare that they have no conflicts of interest.

\section{Supplementary Materials}

Supplementary Table 1: ingredients of each herb contained in MBSHXD. Supplementary Table 2: known therapeutic targets correspond to the active ingredients. Supplementary Table 3: the target protein corresponds to the gene name from UniPort. Supplementary Table 4: the common targets and corresponding active components. Supplementary Table 5: detailed information of GO and KEGG enrichment analysis for IDD-related targets. Supplementary Table 6: detailed information of GO and KEGG enrichment analysis for common targets. Supplementary Table 7: detailed information of overlapping KEGG pathways. (Supplementary Materials)

\section{References}

[1] D. Hoy, L. March, P. Brooks et al., "The global burden of low back pain: estimates from the global burden of disease 2010 study," Annals of the Rheumatic Diseases, vol. 73, no. 6, pp. 968-974, 2014.

[2] B. I. Martin, R. A. Deyo, S. K. Mirza et al., "Expenditures and health status among adults with back and neck problems," JAMA, vol. 299, no. 6, p. 656, 2008.

[3] Y. Wu, F. Zhang, K. Yang et al., "SymMap: an integrative database of traditional Chinese medicine enhanced by symptom mapping," Nucleic Acids Research, vol. 47, no. D1, pp. D1110-D1117, 2019.

[4] J. Wu, B. Sun, L. Hou et al., "Prospective: evolution of Chinese medicine to treat COVID-19 patients in China," Frontiers in Pharmacology, vol. 11, Article ID 615287, 2020.

[5] X. Zhang, Y. Yang, F. Zhang et al., "Traditional Chinese medicines differentially modulate the gut microbiota based on their nature (Yao-Xing)," Phytomedicine: International 
Journal of Phytotherapy and Phytopharmacology, vol. 85, Article ID 153496, 2021.

[6] W. Liu, Y.-h. Wu, X.-y. Liu, B. Xue, W. Shen, and K. Yang, "Metabolic regulatory and anti-oxidative effects of modified bushen huoxue decoction (补肾活血方) on experimental rabbit model of osteoarthritis," Chinese Journal of Integrative Medicine, vol. 19, no. 6, pp. 459-463, 2013.

[7] L. G. Zhu, P. Zhang, Q. H. Song et al., "Preliminary study of intervention in effect of Bushen Huoxue recipe on calcification of lumbar vertebra cartilage endplate of the aging gerbils," Zhongguo gu Shang = China Journal of Orthopaedics and Traumatology, vol. 30, no. 10, p. 926, 2017.

[8] A. L. Hopkins, "Network pharmacology: the next paradigm in drug discovery," Nature Chemical Biology, vol. 4, no. 11, pp. 682-690, 2008.

[9] S. I. Berger and R. Iyengar, "Network analyses in systems pharmacology," Bioinformatics, vol. 25, no. 19, pp. 2466-2472, 2009.

[10] A. L. Hopkins, "Network pharmacology," Nature Biotechnology, vol. 25, no. 10, pp. 1110-1111, 2007.

[11] E. Guney, J. Menche, M. Vidal, and A.-L. Barábasi, "Networkbased in silico drug efficacy screening," Nature Communications, vol. 7, no. 1, p. 10331, 2016.

[12] M. Kibble, N. Saarinen, J. Tang, K. Wennerberg, S. Mäkelä, and T. Aittokallio, "Network pharmacology applications to map the unexplored target space and therapeutic potential of natural products," Natural Product Reports, vol. 32, no. 8, pp. 1249-1266, 2015.

[13] X.-M. Wu and C.-F. Wu, "Network pharmacology: a new approach to unveiling Traditional Chinese Medicine," Chinese Journal of Natural Medicines, vol. 13, no. 1, pp. 1-2, 2015.

[14] Z. Zhou, B. Chen, S. Chen et al., "Applications of network pharmacology in traditional Chinese medicine research," Evidence-based Complementary and Alternative Medicine, vol. 2020, Article ID 1646905, 7 pages, 2020.

[15] S. Li, T. P. Fan, W. Jia, A. Lu, and W. Zhang, "Network pharmacology in traditional Chinese medicine," Evidencebased Complementary and Alternative Medicine, vol. 2014, Article ID 138460, 2 pages, 2014.

[16] N. Choudhary and V. Singh, “A census of P. longum's phytochemicals and their network pharmacological evaluation for identifying novel drug-like molecules against various diseases, with a special focus on neurological disorders," PLoS One, vol. 13, no. 1, Article ID e0191006, 2018.

[17] N. Choudhary, S. Choudhary, A. Kumar, and V. Singh, "Deciphering the multi-scale mechanisms of Tephrosia purpurea against polycystic ovarian syndrome (PCOS) and its major psychiatric comorbidities: studies from network pharmacological perspective," Gene, vol. 773, Article ID 145385, 2021.

[18] D. Shah, M. Gandhi, A. Kumar, N. Cruz-Martins, R. Sharma, and S. Nair, "Current insights into epigenetics, noncoding RNA interactome and clinical pharmacokinetics of dietary polyphenols in cancer chemoprevention," Critical Reviews in Food Science and Nutrition, vol. 1, 2021.

[19] N. Choudhary and V. Singh, "Insights about multi-targeting and synergistic neuromodulators in Ayurvedic herbs against epilepsy: integrated computational studies on drug-target and protein-protein interaction networks," Scientific Reports, vol. 9, no. 1, Article ID 10565, 2019.

[20] D.-h. Zhang, X. Zhang, B. Peng et al., "Network pharmacology suggests biochemical rationale for treating COVID-19 symptoms with a Traditional Chinese Medicine," Communications Biology, vol. 3, no. 1, p. 466, 2020.

[21] J. Ru, P. Li, J. Wang et al., "TCMSP: a database of systems pharmacology for drug discovery from herbal medicines," Journal of Cheminformatics, vol. 6, no. 13, p. 13, 2014.

[22] D. S. Wishart, Y. D. Feunang, A. C. Guo et al., "DrugBank 5.0: a major update to the DrugBank database for 2018," Nucleic Acids Research, vol. 46, no. D1, pp. D1074-D1082, 2018.

[23] "UniProt: a hub for protein information," Nucleic Acids Research, vol. 43, p. D204, 2015.

[24] M. Rebhan, V. Chalifa-Caspi, J. Prilusky, and D. Lancet, "GeneCards: integrating information about genes, proteins and diseases," Trends in Genetics, vol. 13, no. 4, p. 163, 1997.

[25] J. S. Amberger, C. A. Bocchini, F. Schiettecatte, A. F. Scott, and A. Hamosh, "OMIM.org: Online mendelian inheritance in man $\left(\mathrm{OMIM}^{\circledR}\right)$, an online catalog of human genes and genetic disorders," Nucleic Acids Research, vol. 43, p. D789, 2015.

[26] G. Stelzer, N. Rosen, I. Plaschkes et al., "The GeneCards suite: from gene data mining to disease genome sequence analyses," Current protocols in bioinformatics, vol. 54, pp. 1-33, 2016.

[27] P. Shannon, A. Markiel, O. Ozier et al., "Cytoscape: a software environment for integrated models of biomolecular interaction networks," Genome Research, vol. 13, no. 11, pp. 2498-2504, 2003.

[28] G. Yu, L.-G. Wang, Y. Han, and Q.-Y. He, "clusterProfiler: an $\mathrm{R}$ package for comparing biological themes among gene clusters," OMICS: A Journal of Integrative Biology, vol. 16, no. 5, pp. 284-287, 2012.

[29] D. Szklarczyk, A. L. Gable, D. Lyon et al., "STRING v11: protein-protein association networks with increased coverage, supporting functional discovery in genome-wide experimental datasets," Nucleic Acids Research, vol. 47, no. D1, pp. D607-D613, 2019.

[30] C. H. Chin, S. H. Chen, H. H. Wu, C. W. Ho, M. T. Ko, and C. Y. Lin, "cytoHubba: identifying hub objects and subnetworks from complex interactome," BMC Systems Biology, vol. 8, no. 4, p. S11, 2014.

[31] S. Gu, Y. Xue, Y. Zhang et al., "An investigation of the mechanism of rapid relief of ulcerative colitis induced by fiveflavor Sophora flavescens enteric-coated capsules based on network pharmacology," Combinatorial Chemistry \& High Throughput Screening, vol. 23, no. 3, pp. 239-252, 2020.

[32] J. L. Deng, Y. H. Xu, and G. Wang, "Identification of potential crucial genes and key pathways in breast cancer using bioinformatic analysis," Frontiers in Genetics, vol. 10, p. 695, 2019.

[33] O. Trott and A. J. Olson, "AutoDock Vina: improving the speed and accuracy of docking with a new scoring function, efficient optimization, and multithreading," Journal of Computational Chemistry, vol. 31, no. 2, pp. 455-61, 2010.

[34] L. Wang, J. Lin, and W. Li, "Pharmacological mechanism of danggui-sini formula for intervertebral disc degeneration: a network pharmacology study," BioMed Research International, vol. 2021, Article ID 5165075, 12 pages, 2021.

[35] A. B. Dario, M. L. Ferreira, K. M. Refshauge, T. S. Lima, J. R. Ordoñana, and P. H. Ferreira, "The relationship between obesity, low back pain, and lumbar disc degeneration when genetics and the environment are considered: a systematic review of twin studies," The Spine Journal, vol. 15, no. 5, pp. 1106-1117, 2015.

[36] M.-1. Ji, H. Jiang, X.-j. Zhang et al., "Preclinical development of a microRNA-based therapy for intervertebral disc degeneration," Nature Communications, vol. 9, no. 1, p. 5051, 2018. 
[37] M. Minetama, M. Kawakami, M. Teraguchi et al., "Therapeutic advantages of frequent physical therapy sessions for patients with lumbar spinal stenosis," Spine (Phila Pa 1976), vol. 45, no. 11, p. E639, 2020.

[38] L. Zhu, C. Yu, X. Zhang et al., "The treatment of intervertebral disc degeneration using Traditional Chinese Medicine," Journal of Ethnopharmacology, vol. 263, Article ID 113117, 2020.

[39] S.-H. Feng, F. Xie, H.-Y. Yao, G.-B. Wu, X.-Y. Sun, and J. Yang, "The mechanism of Bushen Huoxue decoction in treating intervertebral disc degeneration based on network pharmacology," Annals of Palliative Medicine, vol. 10, no. 4, pp. 3783-3792, 2021.

[40] T. Li, S. Wang, S. Zhang et al., "Evaluation of clinical efficacy of silver-needle warm acupuncture in treating adults with acute low back pain due to lumbosacral disc herniation: study protocol for a randomized controlled trial," Trials, vol. 20, no. 1 , p. 470, 2019.

[41] K. Zhao, M. Chen, T. Liu et al., "Rhizoma drynariae total flavonoids inhibit the inflammatory response and matrix degeneration via MAPK pathway in a rat degenerative cervical intervertebral disc model," Biomedicine \& Pharmacotherapy, vol. 138, Article ID 111466, 2021.

[42] H.-H. Xu, S.-M. Li, R. Xu, L. Fang, H. Xu, and P.-J. Tong, "Predication of the underlying mechanism of Bushenhuoxue formula acting on knee osteoarthritis via network pharmacology-based analyses combined with experimental validation," Journal of Ethnopharmacology, vol. 263, Article ID 113217, 2020.

[43] S. Yang, L. Li, L. Zhu et al., "Bu-Shen-Huo-Xue-Fang modulates nucleus pulposus cell proliferation and extracellular matrix remodeling in intervertebral disk degeneration through miR-483 regulation of Wnt pathway," Journal of Cellular Biochemistry, vol. 120, no. 12, pp. 19318-19329, 2019.

[44] S. Wang, J. Wei, J. Shi et al., "Follistatin-like 1 attenuation suppresses intervertebral disc degeneration in mice through interacting with TNF- $\alpha$ and smad signaling pathway," Oxidative Medicine and Cellular Longevity, vol. 2021, Article ID 6640751, 13 pages, 2021.

[45] H. Cui, X. Du, C. Liu et al., "Visfatin promotes intervertebral disc degeneration by inducing IL-6 expression through the ERK/JNK/p38 signalling pathways," Adipocyte, vol. 10, no. 1, pp. 201-215, 2021.

[46] J. He, R. Xue, S. Li et al., "Identification of the potential molecular targets for human intervertebral disc degeneration based on bioinformatic methods," International Journal of Molecular Medicine, vol. 36, no. 6, pp. 15931600, 2015.

[47] D. Wang, Y. Chen, S. Cao et al., "Cyclic mechanical stretch ameliorates the degeneration of nucleus pulposus cells through promoting the ITGA2/PI3K/AKT signaling pathway," Oxidative Medicine and Cellular Longevity, vol. 2021, Article ID 6699326, 11 pages, 2021.

[48] J. W. Zhan, S. Q. Wang, M. S. Feng et al., "Effects of axial compression and distraction on vascular bud and VEGFA expression in the vertebral endplate of an ex vivo rabbit spinal motion segment culture model," Spine (Phila Pa 1976), vol. 46, no. 7, p. 421, 2021.

[49] Z. Shao, B. Wang, Y. Shi et al., "Senolytic agent Quercetin ameliorates intervertebral disc degeneration via the Nrf2/NF$\kappa \mathrm{B}$ axis," Osteoarthritis and Cartilage, vol. 29, no. 3, pp. 413-422, 2021.

[50] J. Fei, B. Liang, C. Jiang, H. Ni, and L. Wang, "Luteolin inhibits IL- $1 \beta$-induced inflammation in rat chondrocytes and attenuates osteoarthritis progression in a rat modelflammation in rat chondrocytes and attenuates osteoarthritis progression in a rat model," Biomedicine \& Pharmacotherapy, vol. 109, pp. 1586-1592, 2019.

[51] J. Zhu, H. Tang, Z. Zhang et al., "Kaempferol slows intervertebral disc degeneration by modifying LPS-induced osteogenesis/adipogenesis imbalance and inflammation response in BMSCs," International Immunopharmacology, vol. 43, pp. 236-242, 2017.

[52] C. Feng, H. Liu, M. Yang, Y. Zhang, B. Huang, and Y. Zhou, "Disc cell senescence in intervertebral disc degeneration: causes and molecular pathways," Cell Cycle, vol. 15, no. 13, pp. 1674-1684, 2016.

[53] F. Wang, F. Cai, R. Shi, X.-H. Wang, and X.-T. Wu, "Aging and age related stresses: a senescence mechanism of intervertebral disc degeneration," Osteoarthritis and Cartilage, vol. 24, no. 3, pp. 398-408, 2016.

[54] Z. Liao, R. Luo, G. Li et al., "Exosomes from mesenchymal stem cells modulate endoplasmic reticulum stress to protect against nucleus pulposus cell death and ameliorate intervertebral disc degeneration in vivo," Theranostics, vol. 9, no. 14, pp. 4084-4100, 2019.

[55] Y. Wang, R. Zuo, Z. Wang et al., "Kinsenoside ameliorates intervertebral disc degeneration through the activation of AKT-ERK1/2-Nrf2 signaling pathway," Aging, vol. 11, no. 18, pp. 7961-7977, 2019.

[56] Y.Xi, J. Ma, and Y. Chen, "PTEN promotes intervertebral disc degeneration by regulating nucleus pulposus cell behaviors," Cell Biology International, vol. 44, no. 2, pp. 583-592, 2020.

[57] J. Zhang, X. Wang, H. Liu et al., “TNF- $\alpha$ enhances apoptosis by promoting chop expression in nucleus pulposus cells: role of the MAPK and NF- $\kappa$ B pathways," Journal of Orthopaedic Research, vol. 37, no. 3, pp. 697-705, 2019.

[58] L. Miguélez-Rivera, S. Pérez-Castrillo, M. L. GonzálezFernández et al., "Immunomodulation of mesenchymal stem cells in discogenic pain," The Spine Journal, vol. 18, no. 2, p. 330, 2018.

[59] X.-G. Liu, H.-W. Hou, and Y.-L. Liu, "Expression levels of IL17 and TNF- $\alpha$ in degenerated lumbar intervertebral discs and their correlation," Experimental and Therapeutic Medicine, vol. 11, no. 6, pp. 2333-2340, 2016.

[60] G. Wang, K. Huang, Y. Dong et al., "Lycorine suppresses endplate-chondrocyte degeneration and prevents intervertebral disc degeneration by inhibiting NF- $\kappa$ B signalling pathway," Cellular Physiology and Biochemistry, vol. 45, no. 3, pp. 1252-1269, 2018.

[61] S. Illien-Jünger, O. M. Torre, W. F. Kindschuh, X. Chen, D. M. Laudier, and J. C. Iatridis, "AGEs induce ectopic endochondral ossification in intervertebral discs," European Cells and Materials, vol. 32, p. 257, 2016. 